\title{
Kyoto University graduate school of medicine: tradition and modernity harmonized
}

\author{
Masao Mitsuyama
}

Received: 7 May 2009 / Accepted: 13 May 2009 / Published online: 3 June 2009

(C) Springer-Verlag 2009

Keywords History $\cdot$ Kyoto $\cdot$ Medical school

\section{Beginning of the medical school}

The city of Kyoto boasts a proud heritage tracing back to $794 \mathrm{AD}$ which marked the beginning of its reign as the capital of Japan. It remained the capital for more than a thousand years until 1868, when the Meiji emperor proclaimed the "Meiji restoration" and all executive government functions, along with the title of capital, were transferred to Edo (known today as Tokyo). In 1897, roughly three decades after this transition, Kyoto University was formally established as the second Imperial University, followed two years later by the erection of the national medical school. In its 110-year history, Kyoto University has, among other things, made invaluable contributions to the promotion and advancement of the basic sciences. Since its establishment, six scientists from Kyoto University (graduate or faculty member) have been awarded the Nobel Prize in the fields of physics, chemistry and medicine (Hideki Yukawa 1949, Shin-ichiro Tomonaga 1965, Ken-ichi Fukui 1981, Susumu Tonegawa 1987, Ryoji Noyori 2001 and Toshihide Masukawa 2008).

These monumental achievements aside, the Medical School at Kyoto University has a very modest and humble beginning (Fig. 1). It only started with the fundamental departments of anatomy, physiology, medical chemistry,

M. Mitsuyama $(\bowtie)$

Department of Microbiology,

Kyoto University Graduate School of Medicine,

Kyoto, Japan

e-mail: mituyama@mb.med.kyoto-u.ac.jp pathology, pharmacology, hygiene, legal medicine, internal medicine, surgery, psychiatry, obstetrics and gynecology, pediatrics, ophthalmology, and dermatology. In 1919, the total number of departments was expanded to 24 , in accordance with an amended national policy. Among the countless outstanding research carried out prior to World War II, the extensive research carried out by Dr. Hiroshi Fijinami, a professor of pathology, is revered even today for its impact on modern medicine. Prof. Fujinami not only discovered Schistosoma japonicum, the causative parasite of an endemic disease (Katayama disease), but also found that myxosarcoma in chickens can be transmitted by a filterable agent from the lesion. These findings paved the way towards development of the current concept of viral oncogenesis.

\section{Medical school after World War II}

In 1947, after WWII, the Japanese educational system underwent massive reform and with that the Kyoto Imperial University was formally renamed Kyoto University. The establishment of the Graduate School of Medicine followed soon after in 1955, and covered five major subject areas of medicine: physiology, pathology, social medicine, internal medicine, and surgery. Since its inception, each and every single member of the faculty was charged with the responsibility of training and educating both graduate and undergraduate students. While many distinguished faculty members are widely recognized for their pioneering research, some warrant exceptional recognition. Prof. Kozo Okamoto is a monumental example. During his tenure at the depart- 
Fig. 1 Histology laboratory in the Medical School at Kyoto University, ca, 1914

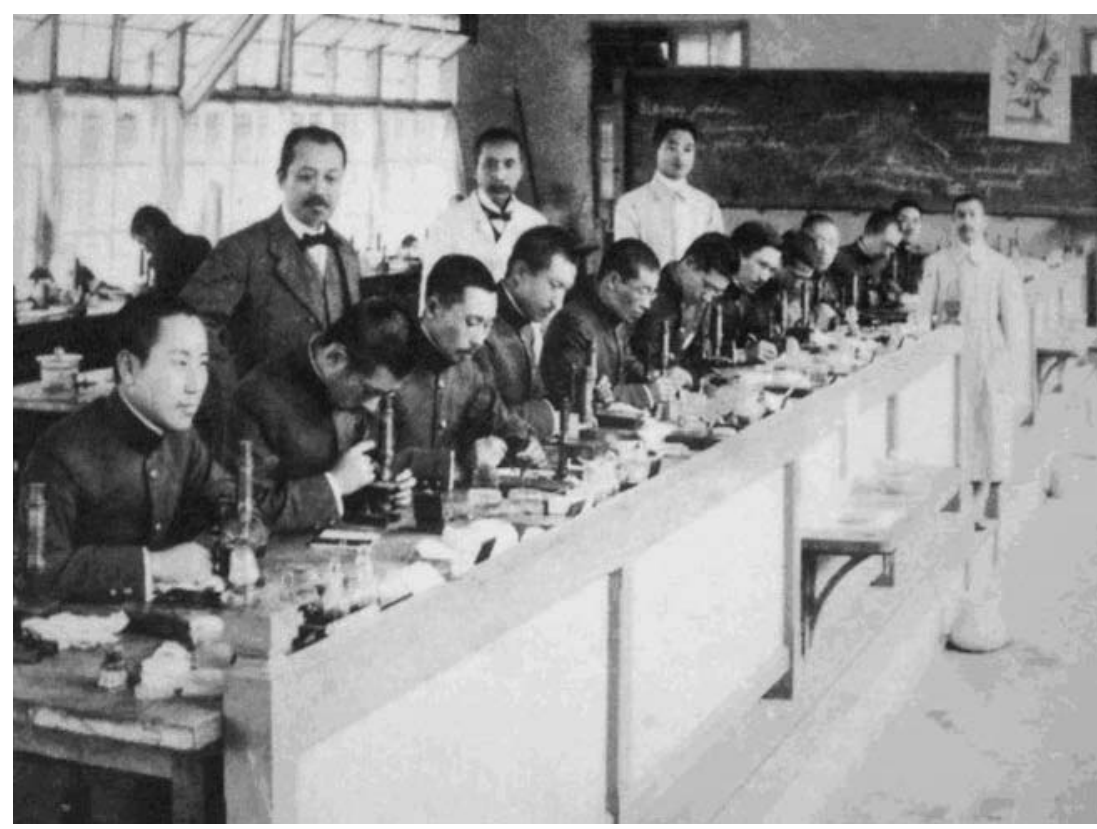

ment of pathology, he established a strain of spontaneously hypertensive rats (SHR) which are actively used in studies of metabolic syndrome today. From the department of medical chemistry, a number of noteworthy biochemists made groundbreaking discoveries in the emerging field of biochemistry and were recognized at an international level for their resounding success. Prof. Osamu Hayaishi discovered "oxygenase" and assumed a central role in the development of molecular enzymology. Prof. Shosaku Numa cloned a series of opioid peptide genes and erected the novel field of molecular neurobiology. Countless biochemists that would eventually become the symbols of progress in their respective fields were trained under the tutelage of these two monumental figures in biochemistry. Among the many are Professor Yasutomi Nishizuka, who discovered protein kinase C; Prof. Tasuku Honjo who discovered AID, an essential molecule involved in class-switch recombination; and Prof. Shigetada Nakanishi who successfully cloned the metabolic glutamate receptor (mGluR) which launched a new era of molecular neuroscience. Prof. Shu Narumiya, the former dean, has also made a lasting mark in modern medical biochemistry. Finally, Porf. Shoichiro Tsukita who passed away at a young age provided significant contributions to the greater understanding of the structural basis of cellular tight junctions by discovering claudin and occuludin. All of these professors have been awarded the Japan Academy Award - the highest and most prestigious academic honor which can be received in Japan. In this manner, the basic research departments at the Graduate School of Medicine at Kyoto University have always had a strong influence on the advancement of the medical sciences in both the foreign and domestic arena.

\section{University Hospital}

For a long period following the foundation of the University Hospital, each clinical department was granted its own building consisting of an outpatient clinic, inpatient ward, physician's office and a laboratory.

Under this previous system, the university hospital was not a consolidated healthcare facility placed under the comfort of one roof, but a compound lined with multiple wooden buildings - each catering to a specific branch of medicine. This style of healthcare prevailed at Kyoto University until 1958 when the construction of an avant-garde centralized hospital building began. This modern university hospital with about 1,300 beds is now composed of 31 clinical divisions, 21 central clinical centers, a pharmacy, and affiliated research centers that would conduct front-line research in aspects such as translational research, clinical trial and management, and medical informatics (Figs. 2 and 3).

Many recent advances in the field of clinical medicine have been lead by our faculty and staff. Prof. Kiyoshi Takatsuki identified a novel clinical entity of adult T-cell leukemia (ATL); Prof. Yorio Hinuma and his colleague discovered the causative agent of ATL, a human retrovirus HTLV-1. Prof. Kazuo Honjou successfully carried out the world's first whole resection of a pancreas. In addition to this, Prof. Koichi Tanaka successfully negotiated one of the first liver transplantation procedures from a live donor in 
Fig. 2 Aerial view of the medical campus, 2005

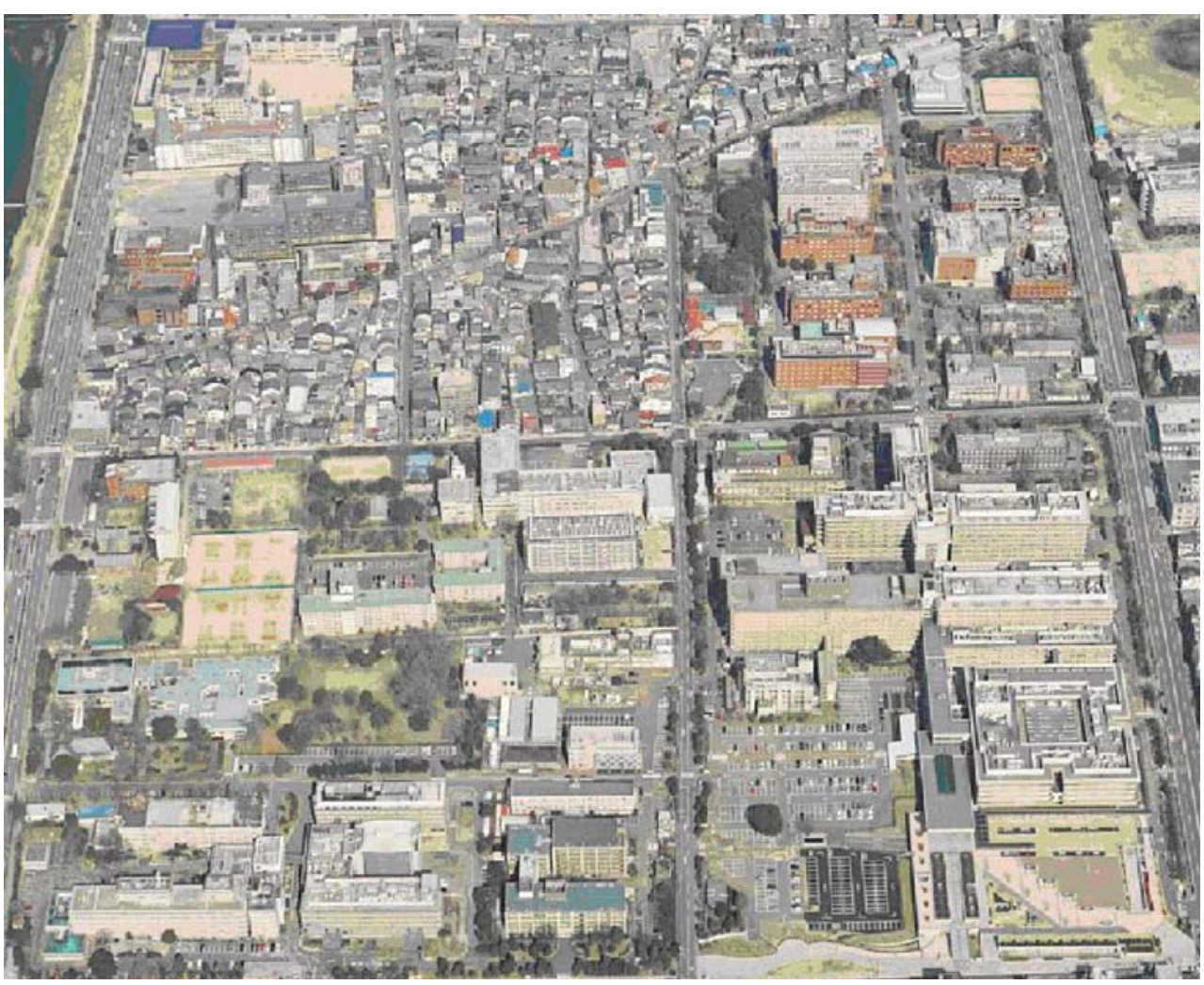

Japan. This is a vital feat considering the lack of organ availability from brain-dead donors in our country. Since then, Kyoto University hospital has assumed an active role in transplantation surgery and has since come to be known as one the world's finest institutions in this field.

\section{The present situation: Education in Medical School}

The undergraduate school provides an intensive 6-year curriculum in medicine. Our students undergo one of the most rigorous selection procedures in the world and only 100 of the most highly competitive candidates are accepted. For the first 18 months, students are required to achieve at least 54 credits in the liberal arts (including foreign languages). Next, in the latter half of their second year, students are introduced to anatomy and histology. The third-year curriculum requires three introductory courses (medical biology, radiation biology and medical informatics literacy), ten lectures in core subjects (histology, gross human anatomy, embryology, molecular cell biology, physiology, neuroscience, etc.) and nine lectures in advanced subjects (pathology, microbiology, immunology, pharmacology, social medicine, etc.). These courses are mandatory and students who fail to obtain passing marks in these subjects may not advance to the next level. Those who move on to their fourth year curricula are exposed to

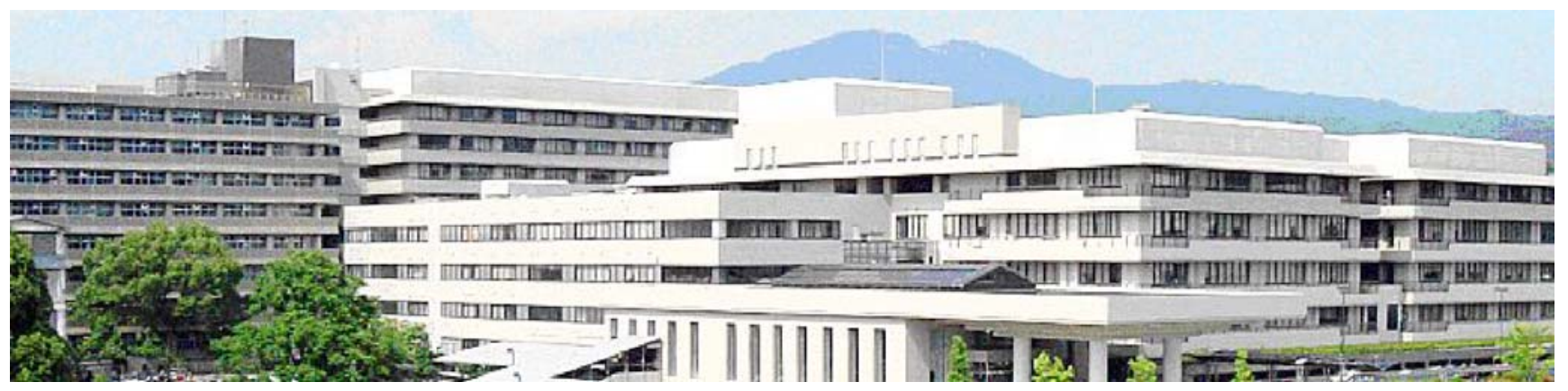

Fig. 3 University Hospital, 2008 
coursework in 27 clinical related contexts which include cardiovascular medicine, hematology, respiratory diseases and gastroenterology.

Traditional classroom instruction has its limits, and in order to promote the acquisition of sound medical knowledge, the first semester for fourth year students are cleared of any mandatory coursework. Instead, students are encouraged to seek brief apprenticeships at active laboratories within Kyoto University, or at any other domestic or foreign academic institutions. This free-semester system provides the ideal setting for each student to gain first-hand exposure in medical science and research. Students who take an active interest in basic research via this system are given the option of switching tracks midstream to an MD/ $\mathrm{PhD}$ course. Those interested in entering the $\mathrm{MD} / \mathrm{PhD}$ course take an entrance examination at the end of their fourth years and, should they pass, enter the Graduate School of Medicine to conduct $\mathrm{PhD}$ research for a period of 4 years. After completion of the $\mathrm{PhD}$ course, the students return to their fifth year of medical school. Upon completion of the medical school curriculum, they are granted an MD. In 2009, two students are in the $\mathrm{MD} / \mathrm{PhD}$ program in basic pathology and cell biology.

For students who continue with the standard - and not $\mathrm{MD} / \mathrm{PhD}$ - course of medical school, the end of the fourth year of medical school marks a defining moment. They must successfully negotiate two major assessments: the CBT and OSCE. The CBT, or computer-based test, is designed for the sole purpose of measuring basic knowledge in medicine. Conversely, the OSCE (objective structured clinical examination) is implemented to gauge the students' aptitude in fundamental clinical skills. Students who pass the CBT and OSCE proceed to their fifth year of medical school. Here, students with demonstrated clinical skills and medical knowledge are divided into small groups. In these groups, the students will perform clinical rounds in the hospital under the guidance of a seasoned staff physician. Most of the major clinical subjects are mandatory; however, elective courses are also offered. The emphasis now is placed on fostering physician scientists and clinical doctors with a wide range of knowledge and interests. The knowledge and skills honed during the 6-year curriculum are put to a final test in what is known as the graduation examination. This is different from the licensing examination and students who pass this examination formally graduate from medical school.

\section{The present: Education in the Graduate School of Medicine}

The scope and frame of the graduate school far surpasses that of the medical school. Kyoto University has 13 affiliated research institutes and some of these are deeply involved in medical science. Some of our more recent accolades include the world's first establishment of iPS cells by Prof. Shinya Yamanaka at the Institute of Frontier Medical Sciences (IFMS), and the discovery of HTLV-1 at the Institute for Virus Research (IVR). The Graduate School of Medicine now consists of over 150 independent laboratories headed by full professors including most of the divisions at IFMS, IVR and some laboratories from the institute for Chemical Research and the Research Reactor institute. In the year 2000, the School of Public Health was founded and has since been integrated into the Graduate School of Medicine. Similarly, the School of Health Sciences has also joined the ranks of the graduate school.

The $\mathrm{PhD}$ course at the Kyoto University Graduate School of Medicine has a diverse background with regard its proportion of international students and researchers. The student population for the graduate school is 700 , of which more than 100 are accounted for by foreign students from countries such as China, Thailand, Bangladesh, Korea, Myanmar, Vietnam, USA, Germany, France, and Nigeria. Graduate students are assigned to and carry out $\mathrm{PhD}$ research at any one of the more than 150 laboratories actively conducting research at the Graduate School. While the students are required to obtain a minimum amount of credits in required coursework, they are mainly assessed by the publication of original articles in international academic journals of high standing and recognition. Numerous papers have been published by our promising graduate students in leading journals such as Nature, Cell, Science and PNAS.

More recently, the "career path unit" was established where outstanding postdoctoral fellows or young faculty members can be promoted to the position of Associate Professor. Those who are selected by a committee are granted a research stipend, independent laboratory space and a couple of laboratory technicians as principal investigator (PI). In the last 5 years, five such PIs have been promoted to the status of full professors in Japan.

To facilitate inter-specialty collaboration among individual laboratories, we have initiated several projects supported by generous sums provided by national funding. The COE (Center of Excellence) project is a prime example and promotes, among many other things, collaboration in the field of animal disease models and regenerative medicine (tissue engineering). Collaborative studies on lifestyle disease and cancer by the recently established School of Public Health, Translational Research Center, and Human Genome Research Center are now also underway and are expected to lead pave a path for the advancement of the clinical science not only in Japan but on the international stage as well. 


\section{Conclusion}

Kyoto University Medical School, one of Japan's oldest and most revered institutions, has produced a great number of leading physician-scientists and basic medical scientists. Training the next generation of leaders in the medical sciences will always be our responsibility, but another noteworthy endeavor that is implied in our global standing is the cultivation and establishment of a new era of medical science and clinical medicine; hence the distinction in the manner of reference to our institution. We refer to our institution as the Graduate School of Medicine and not the School of Medicine due to our inherent duty to perform advanced medical research for the betterment of mankind. 